\title{
To Investigate the Effect of Targeted Overdilation in Balloon-Expandable Stents
}

\author{
Jennifer Wong \\ Elliot Backer \\ Joseph C Keenan \\ Huseyin Erhan Dincer \\ Roy Joseph Cho (ID
}

Interventional Pulmonology Division, Department of Pulmonary, Allergy, Critical Care and Sleep Medicine, University of Minnesota, Minneapolis, MN, USA
Correspondence: Roy Joseph Cho

Tel + | 612-626-2637

Email choxx548@umn.edu
Purpose: Balloon-expandable stents are commonly used for the treatment of tracheobronchial strictures. We routinely perform targeted overdilation of these stents $1-2 \mathrm{~mm}$ on initial deployment to prevent stent migration or allow foreshortening to target airway caliber; however, specific data on the effect of targeted overdilation is unknown.

Patients and Methods: We used three iCAST stents $(6 \times 22 \mathrm{~mm}, 7 \times 22 \mathrm{~mm}$, and $10 \times 38 \mathrm{~mm})$ to perform the study. We had two sets of each size to average our results. Targeted overdilation was accomplished with Merit Elation balloons.

Results: The $6 \times 22$ and $7 \times 22$ stent OD increased from 6 to $11.4 \mathrm{~mm}$ and 7 to $11.6 \mathrm{~mm}$. The $10 \times 38$ stent demonstrated minimal OD change with overdilation (OD change of 10.4 to $12.2 \mathrm{~mm})$. All stents demonstrated significant foreshortening with overdilation (20.2 to $5.65 \mathrm{~mm}, 19.4$ to $6 \mathrm{~mm}$, and 30.9 to $10.2 \mathrm{~mm}$ for $6 \times 22,7 \times 22$, and $10 \times 38$, respectively). The breakpoint was seen at near twice the stated stent OD $(13.5 \mathrm{~mm}, 15 \mathrm{~mm}$, and $15 \mathrm{~mm}$ with $6 \times 22,7 \times 22$ and $10 \times 38$, respectively).

Conclusion: We have demonstrated that iCAST stents can increase their OD with subsequent foreshortening during targeted overdilation. This data can help facilitate decisions when selecting a particular iCAST stent for a specific airway application. Additionally, we have highlighted that balloon inflation diameter does not correspond to the actual stent OD during deployment. We believe that this data offers practical information for end-users of this stent type and additional data will be needed to corroborate our findings.

Keywords: airway stenosis, tracheobronchial disease, interventional pulmonary, airway stent, balloon expandable stent, airway balloon dilation

\section{Introduction}

Innovation in technology and interventional pulmonology techniques have paved the way for parallel advancement of airway stents. ${ }^{1,2}$ The iCAST (Atrium Medical Corporation, Merrimack, New Hampshire) stent; in particular, is a balloon expandable covered stent and FDA cleared for the treatment of tracheobronchial strictures. These stents are commonly placed by interventional pulmonologists in the US for central airway obstruction and lobar salvage. ${ }^{3,4}$ The main advantages of these stents lie within its ease of deployment, increased flexibility and greater radial strength. The stent is constructed from 316L stainless steel and is fully covered with two layers of polytetrafluoroethylene (PTFE). The stent and catheter assembly pass through a $2.8 \mathrm{~mm}$ working channel of a bronchoscope, positioned using direct visualization, and deployed when the balloon is fully inflated. Per the manufacturer, the balloon can be inflated to a nominal or burst pressure ( 8 and 12 ATM; respectively) which correspond to the stent's final outer diameter (OD) and length. 
However, we routinely dilate $1-2 \mathrm{~mm}$ past the proposed specifications to reduce the risk of stent migration (ie, in varying lobar airway diameters), allow some foreshortening to target airway caliber, and prevent jailing off another lobar segment when a shorter length is needed. Unlike stents constructed from nitinol, the steel struts allow for overdilation and increase in stent OD with subsequent foreshortening. Currently, the dimensions and characteristics of overdilating this stent is unknown; therefore, the aim of this study is to provide technical information on the axial-to-length relationship and break point of the PTFE following overdilation.

\section{Materials and Methods}

This in vitro study was conducted in the interventional pulmonary airway lab at the University of Minnesota, Minneapolis. The human subjects research committee of the local institutional review board was not contacted given that the study did not involve humans, animal or HIPAA-related information.

The most commonly used iCAST stents (diameter $\times$ length, $\mathrm{mm}$ ) for airway application at our institution include $6 \times 22 \mathrm{~mm}, 7 \times 22 \mathrm{~mm}$ and $10 \times 38 \mathrm{~mm}$; therefore, these sizes were selected for this study. We had two sets of each size with results shown as an average. The $6 \times 22 \mathrm{~mm}$ and $7 \times 22 \mathrm{~mm}$ stents were dilated using an $8-9-10 \mathrm{~mm}$ ( $3 \mathrm{~cm}$ length) and $12-13.5-15 \mathrm{~mm}(3 \mathrm{~cm}$ length) Elation balloons (Merit Medical, Jordan, Utah). The airway balloons were positioned within the stent; whereby, the two radiopaque markers lined up at the proximal and distal edge of the stent. Once the position was secured, we filled the balloon using a saline-filled inflation device (Endotek BIG60, Merit Medical, Jordan, Utah) to the specified diameters of this study. The stent diameter was measured after each inflation using the outerdiameter $(\mathrm{mm})$. The 10 $\times 38$ stent was dilated using 10-11-12mm (3cm length), $12-13.5-15 \mathrm{~mm}$ ( $3 \mathrm{~cm}$ length) and $18-19-20 \mathrm{~mm}(5.5 \mathrm{~cm}$ length) Elation balloons. After an inflation time of $60 \mathrm{sec}$, the balloon was deflated and the stent was removed. The stent OD and length were measured and recorded using digital calipers $(\mathrm{mm})$. The measurements taken from two 6 $\times 22$ and $7 \times 22$ are resulted as mean values. Additionally, we noted the diameter that resulted in the break point of the PTFE (Figure 1). The break point for the PTFE was defined as the initial visualization of the stainless steel through the PTFE coating.

Descriptive statistics (means and standard deviations for continuous variables) were used to summarize the

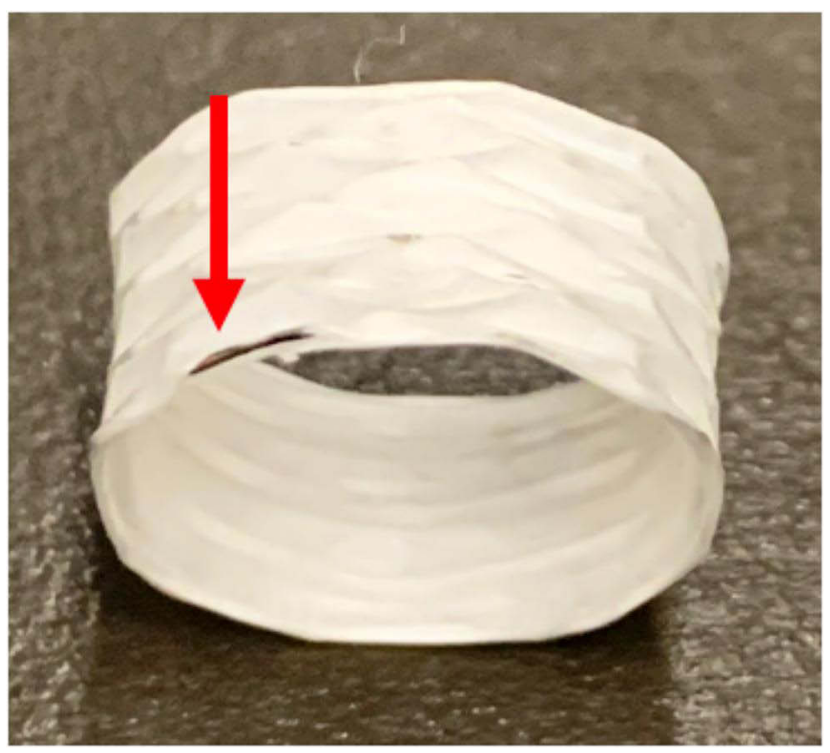

Figure I Stent break point. Red arrow points to the steel strut visualized through the PTFE.

study measurements. All analyses were carried out using Microsoft Excel v16.34 (Redmon, Washington).

\section{Results}

We were able to test all stent sizes and achieved the breakpoint for this study (see Tables 1-3). The baseline OD at the burst pressure of the stents were consistent with the manufacturer specifications; however, the actual lengths were shorter $(20.2 \mathrm{~mm}$ and $30.9 \mathrm{~mm}$ for $6,7 \times$ $22 \mathrm{~mm}$ and $10 \times 38 \mathrm{~mm}$, respectively).

The $6 \times 22$ and $7 \times 22$ stent OD increased from 6 to $11.4 \mathrm{~mm}$ and 7 to $11.6 \mathrm{~mm}$ prior to breakpoint, respectively (Figure 2). All stents demonstrated significant foreshortening with overdilation (20.2 to $5.65 \mathrm{~mm}, 19.4$ to $6 \mathrm{~mm}$, and

Table I Targeted Overdilation of $6 \times 22$ iCAST Stent

\begin{tabular}{|l|l|l|}
\hline $\begin{array}{l}\text { Balloon Diameter } \\
(\mathbf{m m})\end{array}$ & $\begin{array}{l}\text { Stent OD } \\
(\mathbf{m m})\end{array}$ & $\begin{array}{l}\text { Stent Length } \\
(\mathbf{m m})\end{array}$ \\
\hline $6.0^{*}$ & $6.2^{*}$ & $20.2^{*}$ \\
8.0 & 6.2 & 18.6 \\
9.0 & 7.1 & 18.4 \\
10.0 & 7.9 & 17.7 \\
11.0 & 9.2 & 16.2 \\
12.0 & 9.4 & 14.6 \\
$13.5^{* *}$ & $11.2^{* *}$ & $13.2^{* *}$ \\
15.0 & 11.4 & 11.6 \\
20.0 & 11.4 & 5.7 \\
\hline
\end{tabular}

Notes: Diameter achieved using $8-9-10 \mathrm{~mm}$ and $12-13.5-15 \mathrm{~mm}$ balloons. *Burst pressure dimensions per manufacturer. $* *$ Break point diameter.

Abbreviation: OD, outer diameter. 
Table 2 Targeted Overdilation of $7 \times 22$ iCAST Stent

\begin{tabular}{|l|l|l|}
\hline $\begin{array}{l}\text { Balloon Diameter } \\
(\mathbf{m m})\end{array}$ & $\begin{array}{l}\text { Stent OD } \\
(\mathbf{m m})\end{array}$ & $\begin{array}{l}\text { Stent Length } \\
(\mathbf{m m})\end{array}$ \\
\hline $7.0^{*}$ & $7.3^{*}$ & 19.4 \\
8.0 & 7.4 & 19.0 \\
9.0 & 7.6 & 18.6 \\
10.0 & 7.6 & 18.3 \\
11.0 & 9.4 & 16.2 \\
12.0 & 9.5 & 15.7 \\
13.5 & 10.9 & 9.9 \\
$15.0 * *$ & $11.3 * *$ & $7.0 * *$ \\
20.0 & 11.6 & 6.0 \\
\hline
\end{tabular}

Notes: Diameter achieved using 8-9-10 $\mathrm{mm}$ and $12-13.5-15 \mathrm{~mm}$ balloons. *Burst pressure dimensions per manufacturer. $* *$ Break point diameter.

Abbreviation: OD, outer diameter.

Table 3 Targeted Overdilation of $10 \times 38$ iCAST Stent

\begin{tabular}{|l|l|l|}
\hline $\begin{array}{l}\text { Balloon Diameter } \\
(\mathbf{m m})\end{array}$ & $\begin{array}{l}\text { Stent OD } \\
(\mathbf{m m})\end{array}$ & $\begin{array}{l}\text { Stent Length } \\
\mathbf{( m m})\end{array}$ \\
\hline $10.0^{*}$ & $10.4^{*}$ & $30.9 *$ \\
11.0 & 9.9 & 13.9 \\
12.0 & 10.2 & 32.2 \\
13.5 & 12.5 & 31.4 \\
$15.0^{* *}$ & $12.9^{* *}$ & $31.0^{* *}$ \\
20.0 & 12.0 & 30.0 \\
\hline
\end{tabular}

Notes: Diameter achieved using 8-9-10mm, 12-13.5-15mm, and 18-19-20mm balloons. *Burst pressure dimensions per manufacturer. **Beak point diameter. Abbreviation: OD, outer diameter.

30.9 to $10.2 \mathrm{~mm}$ for $6 \times 22,7 \times 22$, and $10 \times 38$, respectively). However, the $10 \times 38$ stent demonstrated minimal OD change with overdilation (OD change of 10.4 to $12.2 \mathrm{~mm})$. The breakpoint was observed near twice the stated stent OD $(13.5 \mathrm{~mm}, 15 \mathrm{~mm}$, and $15 \mathrm{~mm}$ with $6 \times$ $22,7 \times 22$ and $10 \times 38$, respectively).

\section{Discussion}

Over the past 20 years, there has been significant advancement in the discipline of interventional pulmonary with parallel growth of medical devices in this space. In particular, there are a numerous selection of tracheobronchial stents with unique insertion techniques available in the treatment of tracheobronchial disease. In severe or refractory tracheobronchial disease, airway stenting can be considered; however, complications including mucous impaction, granulation tissue formation, stent migration and risk of infection must be acknowledged prior to the use of these devices. Additionally, all stents have their limitations and careful patient selection remains paramount when used in combination with endoscopic interventions. ${ }^{5}$ The purpose of this study was to provide practical data during targeted overdilation of iCAST stents which remain the only balloon expandable option for airway application.

In this study, we demonstrated the capability of these stents during targeted overdilation and subsequent foreshortening over the series of dilations. This data also highlights the dog-bone phenomenon; in addition, to discrepancies between different diameter stents of a given length which are unrecognized by interventional pulmonologists. We chose the "breakpoint" as our limit to overdilation given that it disrupts the PTFE coating which is largely recognized as stent fracture. In this study, the breakpoint occurred at approximately two times the original diameter for these given stents. It should be noted, we do not recommend targeted overdilation past the breakpoint given potential struct fracture and injury to smaller airways. Although this stent is not FDA approved for application in children, overdilation in this population could cause more airway injury due to the smaller caliber of the airways. Alternatively, the larger $10 \times 38 \mathrm{~mm}$ stent did not demonstrate much change in OD; however, did have significant foreshortening with overdilation. Notably, the $10 \times 38 \mathrm{~mm}$ stent was prone to reaching the break point at a lower overdilation diameter compared with the smaller stents (see Figure 2).

We discovered that the actual length of each stent was shorter than stated in the manufacturer specifications. The reason for this is because each varying stent size (OD) at a given length are loaded onto a larger diameter balloon accordingly. Therefore, the smallest OD stent at a given length reflects the most accurate diameter and length per the manufacturer. Another interesting phenomenon was the discrepancy between balloon dilation and actual stent OD. The balloon dilation measurement was always greater compared to the actual stent OD. This relationship is best seen in Figure 3. Since the length of the balloon was longer than the stent, the balloon was able to continue dilating in the non-covered stent area (distal and proximal end) even when the maximal OD of the stent was reached. We call this the "dog-bone" phenomenon. This phenomenon occurs at larger diameters (see Tables 1-3); whereby, 


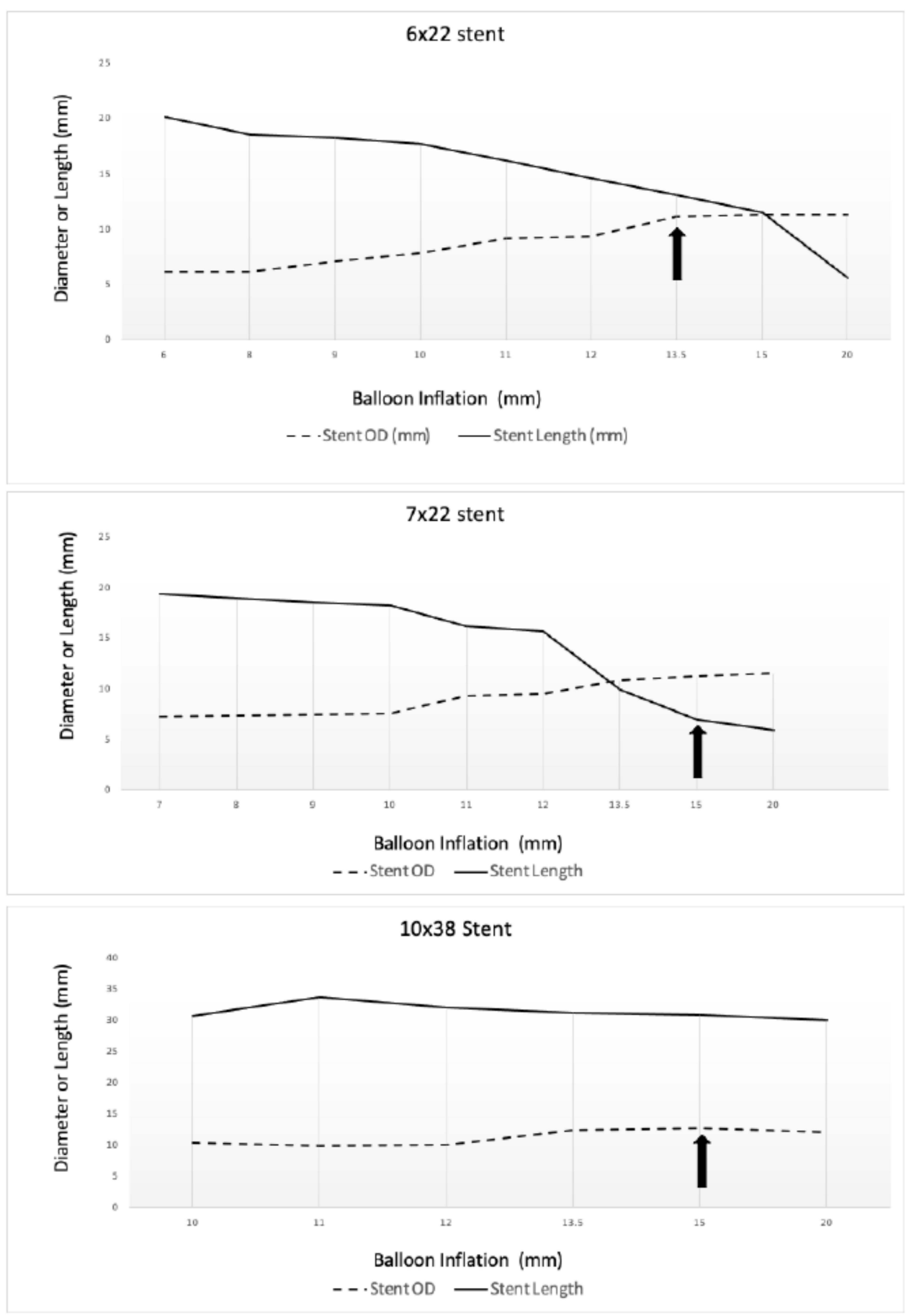

Figure 2 Over dilation of iCAST stents. Above graphically illustrates the effect of over dilation on stent outer diameter (OD) and length. The 6 and $7 \times 22$ stents show significant contraction at $12 \mathrm{~mm}$ dilation. The $10 \times 38$ stent did not show significant contraction during over dilation. All stent appeared to have a break point at $15 \mathrm{~mm}$ dilation.

the overdilation tends to plateau. We believe the dogbone phenomenon is maximal at the breakpoint limit of these stents. This information is important because the user cannot rely on the balloon measurements to determine the true stent OD during targeted overdilation.
There are several limitations to this study. Ideally, we would have had multiple repeated measurements however we were limited to two sets of each stent size; therefore, our results may be subject to sample size error. Second, our inflation balloons were longer than the stent length; 

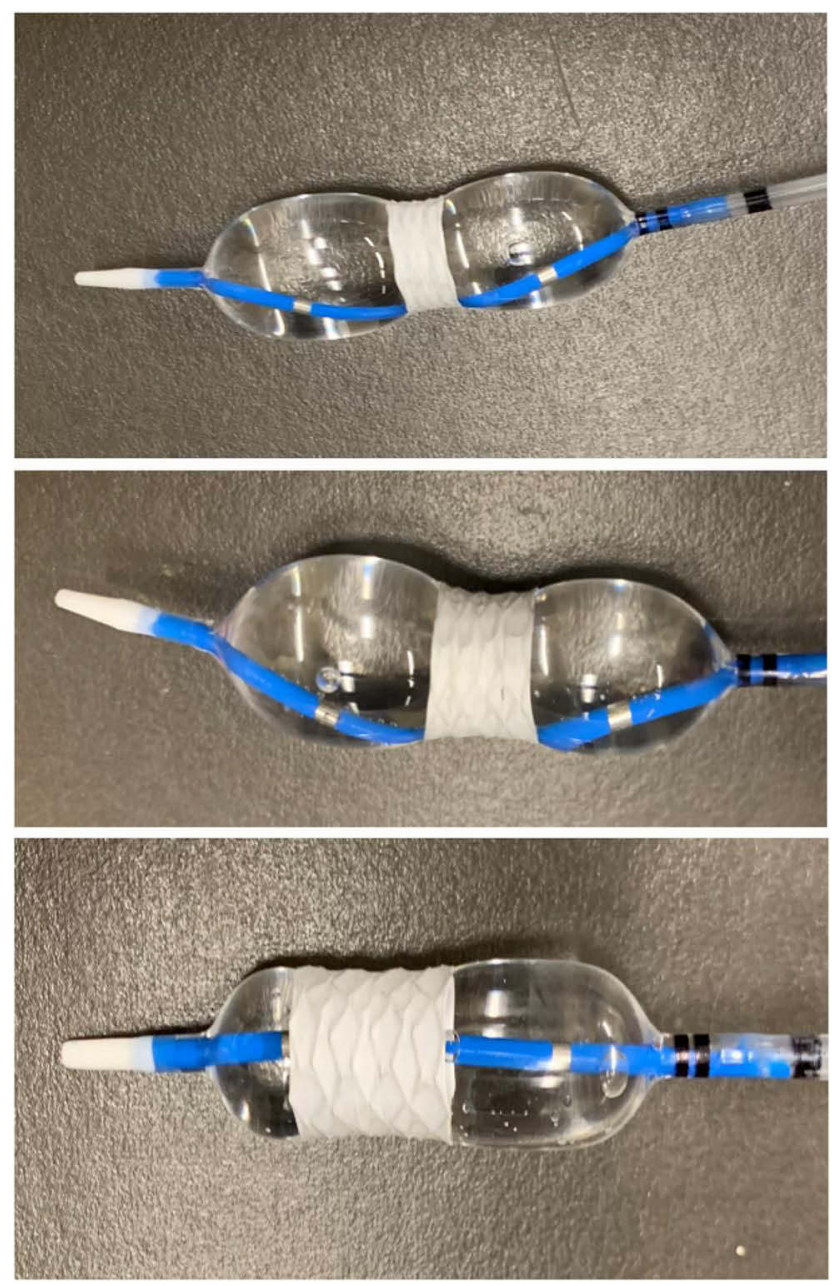

Figure 3 Change in length and outer diameter (OD) during serial targeted overdilation. Above graphically illustrates the effect of overdilation on stent outer OD (dotted line) and length (solid line). The 6 and $7 \times 22$ stents showed significant contraction over the series of dilations unlike the larger $10 \times 38$ stent. All stent appeared to have a break point at two times the original stent diameter (arrow).

therefore, use of balloons shorter than the stent length may offer differing results. Despite these limitations, we believe that this study is noteworthy given that the data is novel with respect to stent dimensions and our distinctive observations of this stent type during overdilation.

\section{Conclusion}

In conclusion, we have demonstrated the characteristics of these stents during targeted overdilation where we highlight the relationship between larger diameters and stent foreshortening. In complex cases, this data could help facilitate stent choice when the optimal size and length for a particular application is unavailable. Additionally, we have highlighted that balloon inflation diameter does not correspond to the actual stent OD during deployment. We believe that this data offers practical information for endusers of this stent type and additional data will be needed to corroborate our findings.

\section{Acknowledgments}

We thank the Atrium Medical Corporation for donating these stents for scientific research.

\section{Disclosure}

The authors report no conflicts of interest in this work.

\section{References}

1. Montgomery WW. T-tube tracheal stent. Arch Otolaryngol. 1965;82 (3):320-321. doi:10.1001/archotol.1965.00760010322023

2. Dumon J-F. A dedicated tracheobronchial stent. Chest. 1990;97 (2):328. doi:10.1378/chest.97.2.328

3. Majid A, Kheir F, Chung J, et al. Covered balloon-expanding stents in airway stenosis. J Bronchology Interv Pulmonol. 2017;24(2):174-177. doi:10.1097/LBR.0000000000000364

4. Sethi S, Gildea TR, Almeida FA, et al. Clinical success stenting distal bronchi for "lobar salvage" in bronchial stenosis. J Bronchology Interv Pulmonol. 2018;25(1):9-16. doi:10.1097/LBR.0000000000000422

5. Ayub A, Al-Ayoubi AM, Bhora FY. Stents for airway strictures: selection and results. $J$ Thorac Dis. 2017;9(S2):S116-S121. doi:10.21037/jtd.2017.01.56
Medical Devices: Evidence and Research

\section{Publish your work in this journal}

Medical Devices: Evidence and Research is an international, peerreviewed, open access journal that focuses on the evidence, technology, research, and expert opinion supporting the use and application of medical devices in the diagnosis, monitoring, treatment and management of clinical conditions and physiological processes. The identification of novel devices and optimal use of existing devices which will lead to improved clinical outcomes and more effective patient management and safety is a key feature of the journal. The manuscript management system is completely online and includes a very quick and fair peer-review system. Visit http:// www.dovepress.com/testimonials.php to read real quotes from published authors. 